\title{
Dynamic Insertion of Pot3 in AvrPib Prevailing in a Field Rice Blast Population in the Philippines Led to the High Virulence Frequency Against the Resistance Gene Pib in Rice
}

\author{
Toluwase Olukayode, ${ }^{1,2}$ Berlaine Quime, ${ }^{2}$ Yin-Chi Shen, ${ }^{2,3}$ Mary Jeannie Yanoria,2 Suobing Zhang,, ${ }^{2,4}$ \\ Jianyuan Yang, ${ }^{5}$ Xiaoyuan Zhu, ${ }^{5}$ Wei-Chiang Shen, ${ }^{3}$ Andreas von Tiedemann, ${ }^{1}$ and Bo Zhou ${ }^{2, \dagger}$ \\ ${ }^{1}$ Division of Plant Pathology and Crop Protection, Department of Crop Sciences, Faculty of Agricultural Sciences, Georg-August University, \\ Grisebachstraße 6, D-37077 Göttingen, Germany; ${ }^{2}$ International Rice Research Institute, DAPO Box 7777, Metro Manila, Philippines; \\ ${ }^{3}$ Department of Plant Pathology and Microbiology, National Taiwan University, Taipei, 10617 Taiwan; ${ }^{4}$ Provincial Key Laboratory of \\ Agrobiology, Jiangsu Academy of Agricultural Sciences, Nanjing 210014, China; and ${ }^{5}$ Guangdong Provincial Key Laboratory of High \\ Technology for Plant Protection, Plant Protection Research Institute, Guangdong Academy of Agricultural Sciences, Guangzhou, \\ 510640, China
}

Accepted for publication 11 November 2018.

\begin{abstract}
The Magnaporthe oryzae avirulence gene AvrPib is required for the resistance mediated by its cognate resistance gene $P i b$, which has been intensively used in indica rice breeding programs in many Asian countries. However, the sequence diversity of AvrPib among geographically distinct $M$. oryzae populations was recently shown to be increasing. Here, we selected a field population consisting of 248 rice blast isolates collected from a disease hotspot in Philippine for the analysis of $A v r P i b$ haplotypes and their pathogenicity against Pib. We found that all of the isolates were virulent to $P i b$ and each of them contained an insertion of Pot3 transposon in AvrPib. Moreover, Pot3 insertion was detected in different genomic positions, resulting in three different AvrPib haplotypes, designated avrPib-H1 to $\mathrm{H} 3$. We further

belonging to the same lineage often had the same AvrPib haplotype. In contrast, the isolates having the same AvrPib haplotypes did not always belong to the same lineages. Both mating types MAT1-1 and MAT1-2 were identified in the population in Bohol and the latter appeared dominant. On the host side, we found that 32 of 52 released rice varieties in the Philippines contained Pib diagnosed by PCR gene-specific primers and DNA sequencing of gene amplicons, suggesting that it was widely incorporated in different rice varieties. Our study highlights the genetic dynamics of rice blast population at both the AvrPib locus and the genome-wide levels, providing insight into the mechanisms of the mutations in AvrPib leading to the breakdown of Pib-mediated resistance in rice.
\end{abstract} conducted a genome-wide Pot2 fingerprinting analysis by repetitive element palindromic polymerase chain reaction (PCR) and identified seven different lineages out of 47 representative isolates. The isolates
Keywords: AvrPib, haplotype, Pib, Pot3, repetitive element palindromicPCR.
Plants are often challenged by a number of biotic stresses and, thus, have evolved two layers of immunity mediated by transmembrane pattern recognition receptors and the intracellular nucleotide-binding leucine-rich repeat (NB-LRR or NLR) proteins (Cesari et al. 2013). To break the basal plant immunity, pathogens have evolved a repertoire of effectors which interfere with the pattern recognition receptor and, thus, cause disease. In an antagonistic coevolution, plants have evolved the NLR protein to recognize pathogen effectors as a second layer of immunity (Jones and Dangl 2006) and, in return, pathogens manipulate its effector to evade recognition by the host plant. This continuous coevolution between hosts and pathogens has led to the proposition of two evolutionary models (i.e., the arms race and trench warfare)

†Corresponding author: B. Zhou; E-mail: b.zhou@irri.org

Present address of T. Olukayode: Huck Institute of the Life Sciences, The Pennsylvania State University, University Park 16802, U.S.A.

Funding: This work was supported, in part, by grants from the Council of Agriculture in Taiwan, Guangdong Science and Technology International Joint Project (2016A050502030), the SCPRID program of BBSRC, the Bill \& Melinda Gates Foundation, and the Research Program on Rice Agri-food System.

*The $\boldsymbol{e}$-Xtra logo stands for "electronic extra" and indicates that three supplementary tables are published online.

The author(s) declare no conflict of interest.

(C) 2019 The American Phytopathological Society
(Kanzaki et al. 2012; Stahl et al. 1999; Woolhouse et al. 2002). Pathogens need to retain their fitness and, therefore, they evolve toward virulence and virulent strains emerge in the pathogen population. Within the pathogen population, such a virulence allele increases rapidly and, in the end, replaces the avirulent allele (Stergiopoulos et al. 2007). Conversely, the host evolves an antagonistic gene that invalidates the new virulent allele, and this corresponding process repeats over time (Kanzaki et al. 2012; Woolhouse et al. 2002).

Rice blast disease, caused by the filamentous fungal pathogen Magnaporthe oryzae, is the most catastrophic fungal disease in rice production worldwide (Talbot 2003). The genetic-based control strategy remains the most economic and efficient strategy to combat the disease. More than 80 rice blast resistance $(R)$ genes have been identified and approximately 25 of them have been cloned (Fukuoka et al. 2012; Su et al. 2015), many of which have already been introgressed into elite rice varieties (Khush and Jena 2009). All of these $R$ genes encode the highly polymorphic NB-LRR proteins, with the exception of pi21 and Pid2, which encode a proline-rich protein and a B-lectine kinase protein, respectively (Chen et al. 2006, 2014; Fukuoka et al. 2009). Conversely, 11 avirulence (Avr) genes have been characterized to date in the rice blast pathogen, all of which encode secreted proteins of as-yet-unknown function, except $A C E 1$. ACE1 encodes a polyketide and peptide synthase (Böhnert et al. 2004) and is uniquely different from other known Avr genes in that it is not the $A C E 1$ protein itself that is recognized by the host plant but the enzyme product of its secondary metabolite (Valent 
and Khang 2010). AvrPiz-t suppresses basal immunity by affecting flg22-mediated generation of reactive oxygen species (Park et al. 2012). It interferes with E3 ligase activity and promotes protein degradation by interacting with rice RING E3 ligases APIP6 and APIP10 (Park et al. 2012, 2016).

It is evident that resistant varieties are usually defeated by the evolution of virulent strains shortly after deployment mainly caused by the quick evolution of the pathogen. It has been proposed that the frequent evolution toward virulence in $M$. oryzae is mainly due to mutations in Avr genes (Orbach et al. 2000). Rice blast fungus and many other plant pathogens use DNA modification in their Avr gene to suppress or evade host recognition, thereby allowing them to infect a previously resistant plant genotype (Kang et al. 2001; Zhou et al. 2007). Such DNA manipulations include frameshift mutation, insertion of transposon, and point mutations within the Avr gene or its complete loss (Wu et al. 2015; Yoshida et al. 2009). Beyond DNA mutations, alteration of the expression of a corresponding Avr gene by causing mutation in its regulatory region has also been reported as one of the mechanisms pathogens use to evade host recognition (Shan et al. 2004).

The $P i b$ gene identified from the indica rice gene pool belongs to the NBS-LRR class of plant $R$ genes encoding a polypeptide of approximately 1,251 amino acids (Wang et al. 1999). Long before $P i b$ was cloned, it had been deployed by several breeding programs and introgressed into rice cultivars in Philippines, Japan, China, and many other rice-growing regions of the world to confer resistance to rice blast races with the cognate $A v r$ gene (Wang et al. 1999; Zeigler et al. 1997). More recently, $P i b$ together with $P i-k h$ was introgressed into elite varieties through marker-assisted back crossing due to the broad-spectrum resistance of the gene in Malaysia (Tanweer et al. 2015). Varieties carrying the $P i b$ gene have been intensively used in indica rice breeding programs in Asia. For instance, IR 50 contains $P i b$ and a number of successful Chinese cultivars, notably Teqing and Texianzhan25, carry the Pib R gene (Khush and Jena 2009; Zhang et al. 2015). Its cognate gene, AvrPib, has recently been isolated by a map-based cloning method in M. oryzae. It encodes a 75-residue protein, including a signal peptide (Zhang et al. 2015). Phenotyping and genotyping of Chinese isolates revealed four genetic events leading to its loss of function (i.e., transposable element [TE] insertion, segmental deletion, absence, and point mutation) (Zhang et al. 2015).

Identification of the molecular mechanisms of pathogen adaptation and evolution, particularly effector diversification, is important for pathogen-informed crop protection. In this study, we investigated the effectiveness of Pib-mediated resistance in the Philippines and the molecular basis of adaptation of Philippine isolates of $M$. oryzae to Pib. We analyzed the sequence variation in $A v r P i b$ of Philippine field isolates and determined their correlation between the AvrPib genotype and pathotype to the Pib gene. We also investigated the association between AvrPib haplotypes and genome-wide diversity based on DNA fingerprints. The frequency of $P i b$ in released varieties was also investigated to provide insight into the host force driving the evolution of rice blast toward virulence.

\section{MATERIALS AND METHODS}

Fungal isolates, culture, rice cultivars, and pathogenicity assay. Rice-blast-infected leaf samples were collected from a rice blast hotspot in Bohol, the Philippines. In total, 250 isolates were isolated from the leaf samples. Isolates were preserved on filter paper in Petri dishes and stored in a $-20^{\circ} \mathrm{C}$ refrigerator. Stored isolates were revived on prune agar medium and grown for 7 days in a $28^{\circ} \mathrm{C}$ incubator. Revived isolates were subcultured on the prune agar medium for another 7 days and mycelia were scrapped with a sterilized glass slide. Scraped fungal cultures were placed under near UV light for 3 days at $18^{\circ} \mathrm{C}$ to enhance sporulation. A suspension of $5 \times 10^{4}$ conidia $/ \mathrm{ml}$ was made from highly sporulating plates and used for inoculation. Tween $20(600 \mu \mathrm{l})$ was added into 1 liter of autoclaved water for suspension preparation to enhance adhesion of fungal spores on plant leaves. The $P i b$ rice monogenic International Rice Research Institute (IRRI)-bred Blast-resistant line (IRBL)b-B and two susceptible checks, its backcross parent Lijiangxintuanheigu (LTH) and CO39, were used for the pathogenicity assay. Pregerminated seed of IRBLb-B, LTH, and CO39 were sown at 10 seeds per line per row in a rectangular food keeper. Rice seedlings at the three- to four-leaf stage were used for the pathogenicity assay. Spraying inoculation method was employed, one food keeper per isolate. In total, 250 isolates together with the standard differential isolates were pathotyped to Pib. Disease reactions were evaluated for susceptible and resistant phenotypes. The disease reactions were evaluated based on the number of lesions using a standard rice blast disease evaluation scale, where 0 to 2 equals resistant and 3 to 5 equals susceptible (IRRI 2014).

DNA preparation, polymerase chain reaction, and sequencing. Fungal isolates were cultured in liquid media at room temperature with continuous shaking for 1 week, and fungal total DNA was isolated from mycelia using a modified cetyltrimethylammonium bromide (CTAB) method (Saghai-Maroof et al. 1984). Mycelia were harvested by suction pressure and transferred into $2.0-\mu$ l tubes and the cell wall was broken mechanically by grinding with $1,000-\mu \mathrm{l}$ micropipette tips in liquid nitrogen. Afterward, $2 \mathrm{ml}$ of CTAB buffer was added to remove cell components other than the nucleic acids and heated at $65^{\circ} \mathrm{C}$ for $1 \mathrm{~h}$. Chloroform $(600 \mu \mathrm{l})$ was added to each sample, mixed thoroughly, and centrifuged at room temperature at 13,000 rpm for $10 \mathrm{~min}$. Supernatant $(600 \mu \mathrm{l})$ was transferred into $1.5-\mu \mathrm{l}$ tubes, mixed with $400 \mu \mathrm{l}$ of isopropanol, and centrifuged at 13,000 rpm for $5 \mathrm{~min}$. The DNA pellet was washed with $500 \mu \mathrm{l}$ of $70 \%$ ethanol, centrifuged at 13,000 rpm for $3 \mathrm{~min}$, and left to dry in the flow hood for $5 \mathrm{~h}$. After drying, $60 \mu \mathrm{l}$ of polymerase chain reaction (PCR)graded water was added and stored at $-20^{\circ} \mathrm{C}$. Genomic DNAs of rice varieties were isolated from leaf samples of 2-week-old plants using the CTAB method, as described above.

Each PCR was done in a 20- $\mu$ l total reaction volume consisting of $2.0 \mu \mathrm{l}$ of $10 \times$ buffer (Takara Bio Inc.), $1.6 \mu \mathrm{l}$ of dNTPs $(400 \mathrm{mM}$ each), $0.4 \mu \mathrm{l}(10 \mu \mathrm{M})$ of each forward and reverse primers, $14.5 \mu \mathrm{l}$ of distilled water, $0.1 \mu$ l of recombinant Taq DNA polymerase (Takara Bio Inc.), and $1.0 \mu \mathrm{l}(25 \mathrm{ng})$ of template DNA. The PCR for the $A v r P i b$ was performed using the following program: $94^{\circ} \mathrm{C}$ for $2 \mathrm{~min} ; 35$ cycles at $94^{\circ} \mathrm{C}$ for $30 \mathrm{~s}, 58^{\circ} \mathrm{C}$ for $30 \mathrm{~s}$, and $72^{\circ} \mathrm{C}$ for $40 \mathrm{~s}$; and a final extension of $72^{\circ} \mathrm{C}$ for $5 \mathrm{~min}$. All reactions were performed in a G-storm thermal cycler (G-Storm Biotechnology) in a $20-\mu \mathrm{l}$ total volume for detection and $50 \mu \mathrm{l}$ for DNA sequencing. PCR amplicon size was verified by $1 \%$ gel electrophoresis using $100 \mathrm{~V}$ for $30 \mathrm{~min}$ with $2 \mu \mathrm{l}$ of a $1-\mathrm{kb}$ Plus DNA ladder (ThermoFisher Scientific) and was documented with a digital imaging system with Quantity one software (version 4.5.1; BioRad Laboratories). The PCR amplicon was subjected to direct sequencing at Invitrogen (Shanghai). To determine the position of the Pot3 element in AvrPib, the sequence of AvrPib's amplicon using the primer pair $10583 \mathrm{SF}$ and $10583 \mathrm{CR}$ was compared with the one in the avirulent strain (GenBank accession number KM887844). The Pot3 element was identified by homology search using the BLASTN program. The primers used for PCR diagnosis of the AvrPib and Pib genes in rice blast and rice varieties, respectively, are listed in Supplementary Table $S 1$.

Repetitive-element palindromic PCR, cluster analysis, and mating type analysis of field isolates. Genetic diversity study of the rice blast pathogen population was conducted by repetitive-element palindromic (rep)-PCR. rep-PCR was carried out using the primers of POT2-1 and POT2-2, as described previously (GenBank accession number Z33638) (George et al. 1998). Each rep-PCR contained $1.132 \mu \mathrm{M}$ each primer pair, $20 \mathrm{ng}$ of genomic DNA, $0.2 \mathrm{mM}$ each of four dNTPs, and 2.5 U of Ex Taq polymerase in $10 \times$ ex-buffer (Takara Bio Inc.), and was filled to $25 \mu \mathrm{l}$ total volume with sterile nano-pure water. The reaction 
mixture was amplified by initial denaturation at $95^{\circ} \mathrm{C}$ for $2.5 \mathrm{~min} ; 4$ cycles of $1 \mathrm{~min}$ of denaturation at $94^{\circ} \mathrm{C}$, annealing at $62^{\circ} \mathrm{C}$ for $1 \mathrm{~min}$, and extension at $65^{\circ} \mathrm{C}$ for $10 \mathrm{~min}$; followed by 26 cycles of $30 \mathrm{~s}$ of denaturation at $94^{\circ} \mathrm{C}$, annealing at $62^{\circ} \mathrm{C}$ for $1 \mathrm{~min}$, and extension at $65^{\circ} \mathrm{C}$ for $10 \mathrm{~min}$; with a final extension time of $15 \mathrm{~min}$ at $65^{\circ} \mathrm{C}$ in a G-Storm thermal cycler (G-Storm Biotechnology). The DNA fingerprint was profiled based on the patterns generated by repPCR. The pattern was scored based on the presence or absence of a DNA band of a particular size in all isolates tested, which was then converted into binary codes ( 1 and 0 for presence and absence, respectively, of a particular band size). Dice's coefficient was used to determine the similarity matrices and a cluster analysis was done by using the unweighted pair-group method with arithmetic averages method. The similarity matrices and the cluster analysis were done with BioNumerics software (Applied Math Inc.).

A mating type analysis of isolates was performed using MATspecific primers designed from the sequences of MAT1-1 isolate 70-15 and MAT1-2 isolate Y93-245c-2, with the accession numbers XM_003720674.1 and AB372210.1, respectively. The PCR profile used was as described above for AvrPib amplification.

Phylogenetic analysis. DNA sequences of several amplicons obtained with the Pib homolog-specific primers together with the DNA sequence of the Pib locus in Nipponbare and Shuhui498 were analyzed for their genetic relatedness. The sequence alignments were done with Sequencher software, and the neighbor-joining method was used to construct the phylogenetic tree in the same software with a bootstrap of 1,000 replications.

TABLE 1. $P i b$ homologs in different rice lines

\begin{tabular}{llccc}
\hline Rice lines & \multicolumn{1}{c}{ Primers } & $\begin{array}{c}\text { Group of Pib } \\
\text { homologs }\end{array}$ & $\begin{array}{c}\text { Identity to } \\
\text { Pib }(\%)\end{array}$ & Note \\
\hline $1,5,7,16$ & PibF4/PibR7 & GI & 100 & $\begin{array}{c}\text { Identical } \\
\text { to } P i b\end{array}$ \\
$15,42,47$ & PibF4/PibR7 & GII & 92 & $\ldots$ \\
$3,6,14,16$, & & & & \\
$18,25,47$ & PibF4/Pib-CO39-R5 & GIII & 91 & $\ldots$ \\
15,42 & PibF4/Pib-CO39-R5 & GIV & 97 & $\ldots$ \\
30 & PibF4/Pib-CO39-R5 & GV & 92 & $\ldots$ \\
\hline
\end{tabular}

\section{RESULTS}

Rice blast isolates collected in Bohol, Philippines are virulent to Pib. In total, 248 of 250 isolates collected from a broad range of rice varieties (Table 1) at a rice blast disease hotspot in Bohol were pathotyped against six different IRBLs. These isolates showed a varying frequency of avirulence and virulence against these six IRBLs (Fig. 1A). The virulence frequencies of these isolates against IRBL9-W (Pi9), IRBLi-F5 (Pii), IRBL5-M (Pi5), IRBLk-ka (Pik), and IRBLta-K1 (Pita) were 0, 2, 3, 54, and 62\%, respectively, while all isolates were virulent to IRBLb-B (Pib). However, IRBLb-B is resistant to three Pib avirulent isolates (B90002, V86010, and M101-1-2-9-1) (Fig. 1B), as described previously (Telebanco-Yanoria et al. 2008), suggesting that the virulence of these 248 isolates to IRBLb-B was most likely attributable to the loss of function of AvrPib. Based on the pathotyping data, we concluded that the blast population in Bohol was dynamic in virulence spectrum against different IRBLs, and was highly virulent to $P i b$.

Three virulence haplotypes of $A v r P i b$ are present in rice blast isolates in Bohol. To investigate the mechanisms of the virulence of these isolates against $P i b$, the genomic sequence of the AvrPib gene covering its promoter and coding sequence (CDS) was PCR amplified. Three different PCR amplification patterns were observed in the 248 virulent isolates using different primer pairs along the AvrPib sequence. The first pattern was resolved by the primer pair of AvrPib-5F and AvrPib-1R covering the promoter region of AvrPib (Fig. 2A). A PCR amplicon $>2 \mathrm{~kb}$ in size was obtained in 17 isolates whereas an amplicon with an expected size (376 bp in the avirulent isolates) was obtained in the remaining 231 isolates and the avirulent isolate as well (Fig. 2B; Supplementary Table S2). The second pattern was resolved by the primer pair AvrPib-2F and AvrPib-2R covering the promoter and $5^{\prime}$ portion of CDS (Fig. 2A). Similar to the first pattern, 201 isolates produced amplicons with a larger size whereas the other 47 and the avirulent isolates produced an amplicon with an expected size (Fig. 2B). The third pattern was resolved by the primer pair AvrPib-3F and AvrPib-R covering the $3^{\prime}$ portion of CDS (Fig. 2A). An amplicon with a larger size was obtained in 30 isolates whereas one with an expected size was
A

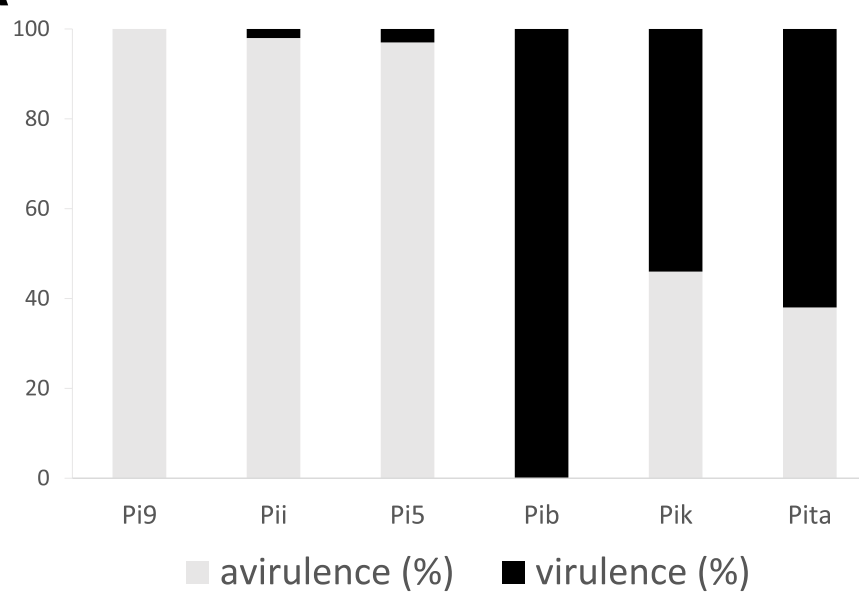

B

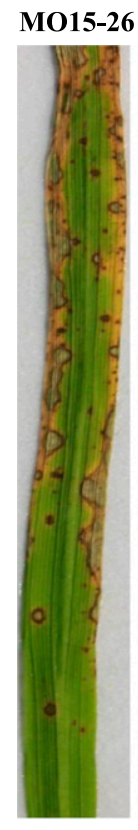

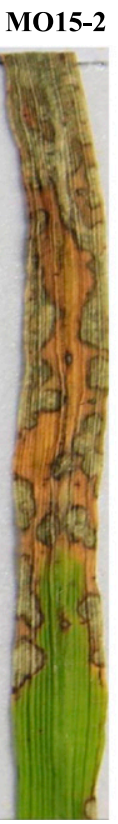
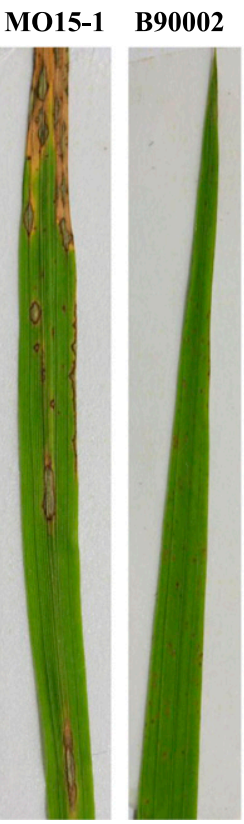

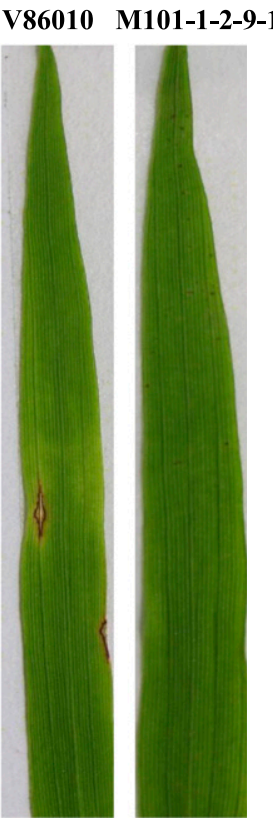

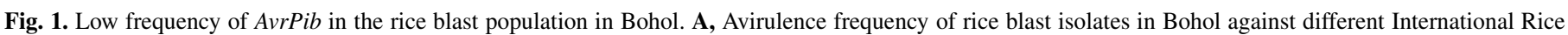

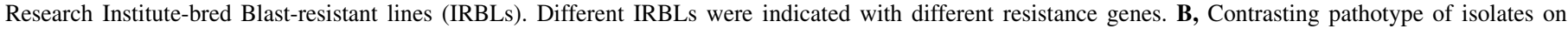

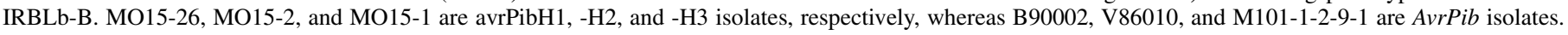


obtained in the other 218 isolates and the avirulent isolates (Fig. 2B). The nature of the amplicons with a larger size was further determined by sequencing. Interestingly, the amplicons from five randomly selected isolates representing each pattern were identical to each other (data not show), suggesting that these three patterns revealed by PCR amplification could represent three different haplotypes of AvrPib, designated herein as avrPib-H1, - H2, and -H3 (Fig. 2B). A sequence analysis further revealed that an insertion of a Pot 3 element $(99 \%$ in overall sequence identity to that of GenBank accession number U60989) was present at the AvrPib region in each of the avrPib-H1, $-\mathrm{H} 2$, and $-\mathrm{H} 3$ isolates. However, the sequence besides the Pot 3 element was identical to the one in the wild-type sequence of $A v r P i b$ (GenBank accession number KM887844), explaining the size discrepancy of the PCR amplicons of these 3 avrPib haplotypes from the one of the avirulent isolate. Pot 3 was present at the positions of $-304,-125$, and $+169 \mathrm{bp}$ in avrPib-H1, $-\mathrm{H} 2$, and $-\mathrm{H} 3$, respectively (Fig. 2A). It is worth noting that none of the isolates contained more than one haplotypes of AvrPib.

The rice blast population in Bohol displays significant genetic diversity. To investigate the mechanisms mediating the spread of $A v r P i b$ haplotypes in field isolates, we studied the genetic diversity of the rice blast population collected in Bohol by Pot2 fingerprinting. In all, 47 isolates consisting of three different AvrPib haplotypes were subjected to DNA fingerprinting by rep-PCR using inverted repeat primers of Pot2. In total, 17 patterns were resolved based on the number and size of DNA bands resolved by rep-PCR (Fig. 3). According to the definition of a lineage in which the patterns share $>70 \%$ similarity to each other by Dice's coefficient analysis, these Pot2 patterns were further clustered into seven lineages (Fig. 3). Within each lineage, all isolates have the same AvrPib haplotypes, suggesting that the isolates having the same $A v r P i b$ haplotypes originated from the same progenitor. However, the isolates having the same AvrPib haplotypes did not always belong to the same lineages. For example, the avrPib-H1 and avrPib-H2 isolates each were scattered into three different lineages, suggesting that these isolates underwent significant sequence divergence from their progenitor after the insertion of Pot3 (Fig. 3 ). Moreover, we found that the isolates having the same AvrPib haplotypes did not always show closer lineage relationships than those having different AvrPib haplotypes. For example, the lineage containing MO15-16, -10 , and -128 isolates having avrPib-H2 shared a closer lineage relationship to the one containing isolates having avrPib-H3 (Fig. 3). This closer lineage relationship was also observed in some isolates having avrPib-H1 and avrPib-H2 haplotypes (Fig. 3). Nonetheless, we noted that the similarity scores connecting these different lineages were relatively low, which may result in an inaccurate relationship among some lineages (Fig. 3).

MAT1-2 is dominant in the rice blast population in Bohol. To investigate the composition of MAT1-1 and -2 isolates in the rice blast population in Bohol, we conducted the mating type analysis of all 248 isolates using specific primers for MAT1-1 and -2. In total, 7 and 245 isolates were MAT1-1 and 1-2, respectively, suggesting that a predominant frequency of MAT1-2 isolates was present in the blast hotspot in Bohol. Intriguingly, four isolates (i.e., MO15-27, -28, -30, and -104) were found to have both mating types, which were further verified by the sequencing of the PCR amplicons. Three AvrPib haplotypes were found in MAT1-2 isolates whereas only avrPib-H1 and -H2 were found in MAT1-1 isolates. Moreover, four MAT1-1 isolates (i.e., MO15-27, -104, -106, and -178) selected for the rep-PCR analysis were found to form a separate lineage and showed a quite distant lineage relationship from other isolates (Fig. 3).

$P i b$ is frequently present in released varieties in the Philippines. To investigate the frequency of $P i b$ in released varieties, we randomly selected 52 varieties released by IRRI and University of the Philippines, Los Baños from 1969 to 2015 (Supplementary Table S3). A sequence analysis between the Pib gene (GenBank accession number AB013448) and its susceptible allele in Nipponbare (position in chromosome 2: 35,102,96035,109,965) (http://signal.salk.edu/cgi-bin/RiceGE) revealed that the $3^{\prime}$ untranslated regions (3'UTR) were highly divergent from each other. Thus, we synthesized several pairs of primers from the $3^{\prime}$ terminus and $3^{\prime}$ UTR of $P i b$ for the diagnosis of its presence in different rice varieties. Three pairs of primers were validated and used for the two-step PCR diagnosis (Fig. 4A). PCR with the primer pair Pib-F3/R5 produced an amplicon at the expected size (approximately $350 \mathrm{bp}$ ) in IRBLb-B and 32 of 52 tested rice varieties (Fig. 4B). Sequencing of this amplicon further revealed that it corresponded to the expected $P i b$ fragment. Unexpectedly, an amplicon at a smaller size (approximately $250 \mathrm{bp}$ ) was observed in 48 tested varieties and the susceptible variety CO39. Nonetheless,

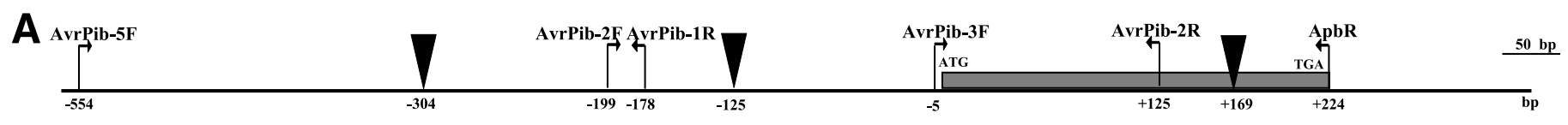

B

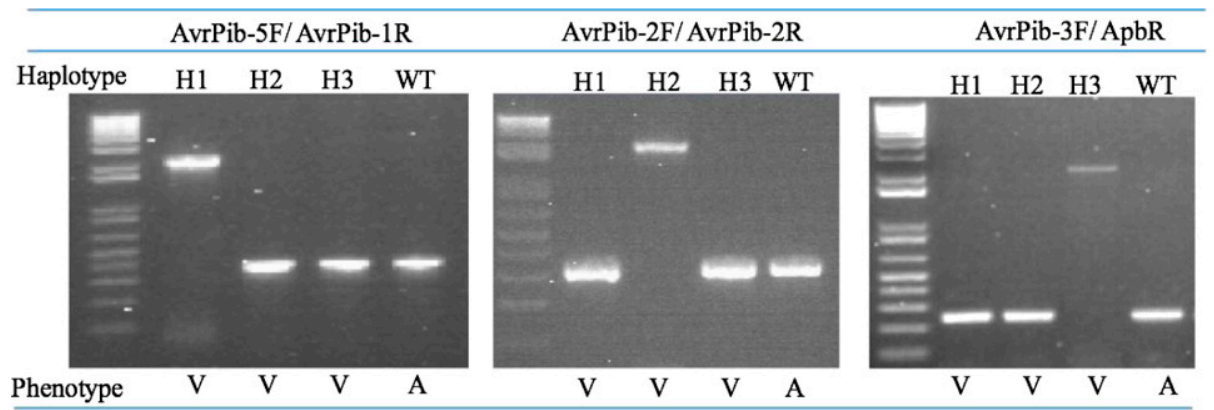

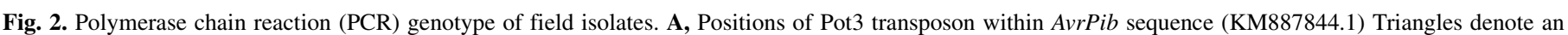

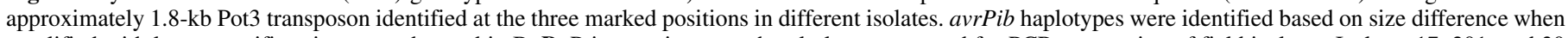

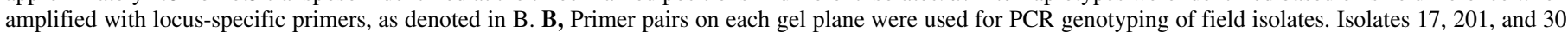

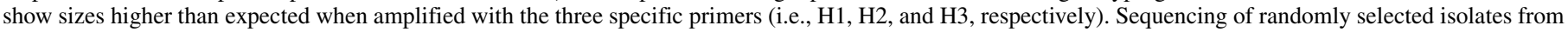

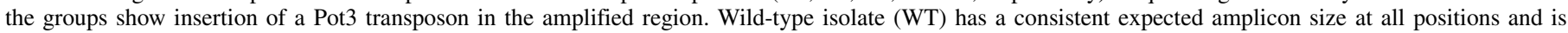

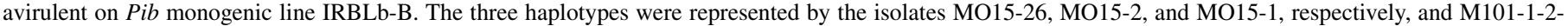
9-1 as the avirulent isolate. 
the sequence of this amplicon turned to be ambiguous, suggesting that the smaller amplicon could be derived from nonspecific amplification. It was found that three rice varieties (i.e., numbers 12 , 23, and 39) did not produce any PCR amplicon, like the two susceptible rice varieties LTH and Nipponbare (Fig. 4). To further determine which varieties contain the $P i b$ gene, two primer pairs specific to $P i b$ and its allele in $\mathrm{CO} 39$ were used for the analysis (Fig. 4A). As expected, all 32 rice varieties producing the 350-bp fragment using Pib-F3/R5 showed the specific amplification with an expected size, as did IRBLb-B using Pib-F4/R7 (Fig. 4B).
However, four other rice varieties (numbers 15, 30, 42, and 47), negative in PCR amplification using Pib-F3/R5, produced the specific amplicon at the similar size by Pib-F4/R7 (Fig. 4B). These four amplicons were then sequenced together with four randomly selected amplicons from lines (numbers 1, 5, 7, and 16). The sequences of all of the amplicons, except the one from number 30 by Pib-F4/R7, were determined and classified into two groups, designated as Pib-GI and -GII (Table 1). It was found that Pib-GI and Pib-GII were identical and had $92 \%$ identity in sequence to Pib, respectively, indicating that those four varieties positive for

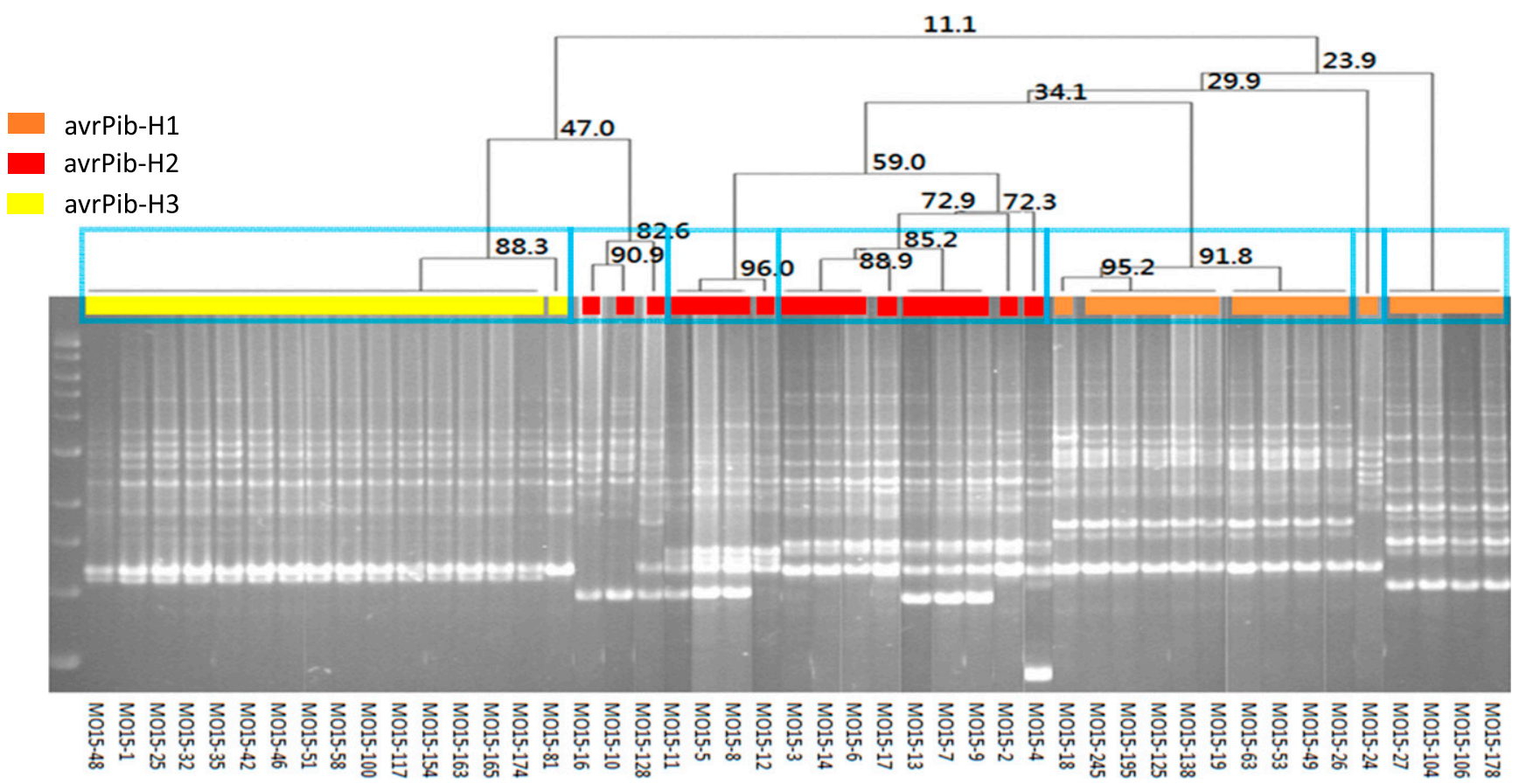

Fig. 3. Repetitive element palindromic polymerase chain reaction (rep-PCR) fingerprinting of Philippine isolates of Magnaporthe oryzae. Forty-seven isolates consisting of three $a v r P i b$ haplotypes were fingerprinted by Pot 2 using inverted repeat primers of Pot 2 . In total, 17 patterns marked in rectangles were identified based on the number and size of DNA bands resolved by rep-PCR. Dice's coefficient was used to calculate similarities between DNA patterns. Seven lineages marked by blue boxes which were defined based on the similarity not less than $70 \%$ between patterns were identified.

A

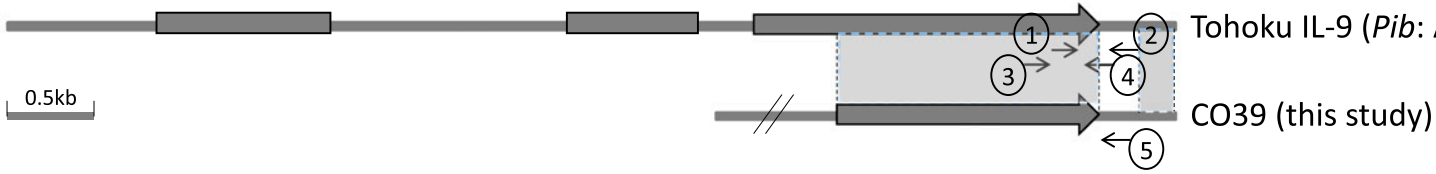

B

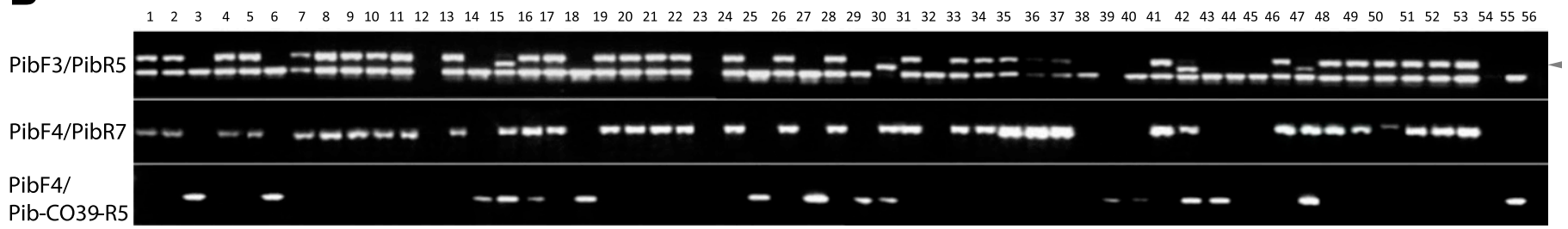

Fig. 4. Polymerase chain reaction (PCR) diagnosis of $P i b$ in rice varieties in the Philippines. A, Schematic diagram of the diagnosis of $P i b$. The primer pair of PibF3 (1) and PibR5 (2) which worked in the Pib donor and CO39 but not in Nipponbare was first used to diagnose the presence of $P i b$ homologs in rice varieties. The primer pair of PibF4 (3) and PibR7 (4) which worked only in the Pib donor but not in CO39 was then used for the diagnosis of the presence of Pib in rice varieties. The primer pair of PibF4 and Pib-CO39-R5 (5) was used for the diagnosis of Pib homologs in CO39. Exons are indicated by rectangles and arrows for the last exon. Regions sharing extremely high sequence similarity ( $>95 \%$ identity on average) are shadowed. The position of the primer is indicated by arrows. B, Three pairs of primers were used to classify the different alleles of Pib in Philippines varieties. The pair PibF3/PibR5 resolved amplicons at the expected size (approximately $350 \mathrm{bp)} \mathrm{in} \mathrm{IRBLb-B} \mathrm{(number} \mathrm{53)} \mathrm{and} 32$ of 52 tested rice lines. Meanwhile, an amplicon at a smaller size (approximately $250 \mathrm{bp)}$ was resolved in 48 tested lines and the susceptible variety CO39. Two more primers specific to Pib and its allele in CO39 were developed. All 32 Pib-positive varieties with PibF3/ PibR5 were also positive with PibF4/PibR7, however. Four varieties (numbers 15, 30, 42, and 47) negative in PCR amplification using PibF3/PibR5 were otherwise positive using PibF4(3)/PibR7(4). Consequently, a total of 15 lines produced specific amplicons, each with an expected size similar to the one from CO39 using PibF4/Pib-CO39-R5(5). 
amplification by both Pib-F3/R5 and Pib-F4/R7 contained Pib. Thus, it is reasonable to speculate that all 32 varieties with expected amplicons by these two primer pairs contained $P i b$. On the other hand, a total of 15 varieties produced specific amplicons of the expected size, similar to the one from CO39 using Pib-F4/ CO39-R5 (Fig. 4B). It is worth noting that only variety number 16 of the 32 varieties positive with $\mathrm{Pib}-\mathrm{F} 3 / \mathrm{R} 5$ produced an amplicon by Pib-F4/CO39-R5. Sequencing of the PCR amplicons by Pib-F3/ CO39-R5 further resulted in the classification of 10 sequences into three groups, designated as Pib-GIII to -GV (Table 1). They shared a significant sequence similarity to $P i b$, varying from 91 to $97 \%$ (Table 1). Nonetheless, none of them were identical to the Pib gene, suggesting that they might be nonfunctional alleles of Pib.

We further investigated the genomic organization of the Pib locus in two rice varieties (Nipponbare and Shuhui498) that have mapbased reference genome sequences. Contrasting to the genomic organization of the Pib locus in Nipponbare, which contained only a single gene $(35,102,960-35,109,965 \mathrm{bp}$ on chromosome 2) (http:// signal.salk.edu/cgi-bin/RiceGE), three $P i b$ homologs designated as Pib-R498-I to III (Fig. 4) were identified at the Pib locus in Shuhui498 (approximately 36,868,779-36,924,798bp on chromosome 2; GenBank accession number CP018158). All of the Pib homologs in different rice varieties identified in this study were then used for building a phylogenetic tree (Fig. 5). In total, nine different Pib homologs were identified (Fig. 5). Moreover, some rice varieties such as numbers $15,16,42$, and 47 contained more than one copy of $P i b$ homologs, which was also observed in Shuhui498 (Table 1). It was found that the Pib allele in typical japonica variety Nipponbare (Pib-NPB), was relatively divergent from those of other indica rice varieties (Fig. 5). Thus, we speculate that the sequence and genomic organization of the Pib locus is highly complex and dynamic in different rice germplasm.

\section{DISCUSSION}

Determination of the effectiveness of a rice $R$ gene based on the information derived from the frequency of its cognate $A v r$ gene in the pathogen population is an informed approach for varietal improvement for resistance against rice blast. $P i b$ is a major gene that was originally identified in indica rice germplasm and was found to confer effective resistance in different regions worldwide (Wang et al. 1999). However, the effectiveness of Pib was documented to be eroding due to the high frequency of virulent isolates in the rice blast population in some regions. Fukuta et al. (2014) reported that more than $60 \%$ of 122 Cambodian isolates were virulent to IRBLb-B and more than $70 \%$ in Hunan province of China (Xing et al. 2017). In this study, we determined the effectiveness of $P i b$ by characterizing the virulence spectrum of a population consisting of 248 field isolates of $M$. oryzae from a hotspot based on pathotyping against the $P i b$ isogenic line and genotyping of AvrPib. All isolates tested were virulent on IRBLb-B, suggesting that $P i b$ has been defeated in the test region. It is worth noting that a blast resistance gene profile in Philippine elite varieties is sparsely known. The molecular diagnosis of 52 historic and advanced varieties showed that $>60 \%$ of tested varieties contained $P i b$. We also traced the introgression of $P i b$ into IRRI varieties back to as early as 1966 in IR8. Our work verified that Pib was extensively used in indica rice breeding in the Philippines since the 1960s (Khush and Jena 2009). The continuous deployment of a particular $R$ gene over a period of time has been proposed to mount selection pressure on the pathogen and strains with the best fitness and reproductive success, favoring the accumulation of the virulent strains in the population and, subsequently, resulting in the loss of effectiveness of the $R$ gene (Jones and Dangl 2006; Kamoun 2007). Thus, we speculate that the wide deployment of $P i b$ over a period of five decades effectuated the positive selection of virulent strains in the population, therefore reducing the effectiveness of Pibmediated resistance. Indeed, IRBLb-B was observed to be highly susceptible in the screening sites in both Bohol and the IRRI experimental station in the Philippines (data not shown).

Different genetic events leading to the loss of function of AvrPib were identified from 300 isolates collected in five different ricegrowing areas in China, out of which TE insertion in various positions accounted for approximately $80 \%$ in the virulent isolates (Zhang et al. 2015). In this study, we found that the AvrPib sequence was disrupted exclusively by a Pot 3 element in all isolates, reiterating the important role of TEs in mediating the loss of function of $A v r$ genes in rice blast (Li et al. 2009; Wu et al. 2015). Moreover, three events of Pot 3 insertion in different positions resulting in the generation of different AvrPib haplotypes were identified, which are unique from those described previously (Zhang et al. 2015) in the Philippine isolates, suggesting that TE insertion in the sequence of $A v r P i b$ could be relatively random and more dynamic. We reason that mutant isolates mediated by the insertion of Pot3 individually at the different positions of AvrPib emerged and gained the virulence to $P i b$, and were further selected by the Pib-harboring varieties and dominated in the population. Given that the isolates dropped within the same lineage were found to have the same AvrPib haplotype, we speculate that clonal reproduction posterior to the different progenitors, each with a unique insertion position of Pot3, could contribute significantly to the build up of the population, as documented in the field rice blast population previously (Levy et al. 1991, 1993). Nonetheless, genetic divergence among isolates having the same AvrPib haplotypes, particularly in avrPib-H1 and - $\mathrm{H} 2$ isolates (Fig. 3), indicates the occurrence of genetic exchange among the isolates, reiterating the finding that $M$. oryzae is not as exclusively clonal as many other fungal species (Chuma et al. 2011; Zeigler et al. 1997).

It is a matter of debate about the mechanisms underlying the genetic recombination in $M$. oryzae. The evidence of parasexual recombination of DNA identified in the rice blast population suggests that parasexual recombination takes place in the field (Zeigler et al. 1997). Chuma et al. (2011) demonstrated the horizontal gene transfer mediated by TEs in asexual clones of the rice blast pathogen. Nevertheless, the extent to which the asexual recombination contributes to the genetic divergence of the rice blast pathogen population is still elusive. Moreover, the sexual cycle was observed in a contemporary population of $M$. oryzae, demonstrating that the rice blast pathogen also reproduces sexually in nature, at least in some regions in Southeast Asia, the center of its origin (Saleh et al. 2012). In this study, we found that both avrPib-H1 and $-\mathrm{H} 2$ isolates contain two mating types, although MAT1-1 is disproportionally low in frequency. Moreover, the MAT1-1 isolates having avrPib-H1 form a separate lineage (Fig. 3), suggesting that genetic recombination took place prior to its separation from other

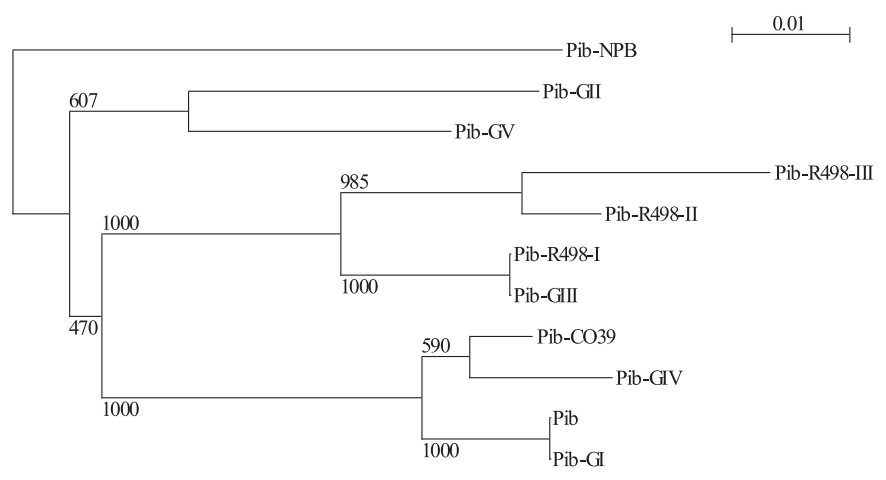

Fig. 5. Phylogenetic relatedness of Pib homologs in different rice varieties. Polymerase chain reaction amplicons in selected rice lines using different primer pairs were sequenced and used for the phylogenetic analysis. The Pib homologs from CO39, Nipponbare, and Shuhui498 (Pib-R498-I to -III) were included in the phylogenetic analysis. Bootstrap values are indicated. 
isolates having the same AvrPib haplotype. Both the presence of two mating types and genetic recombination between isolates imply that sexual recombination could be also involved in the genetic exchange in the rice blast population.

A Southern blotting analysis revealed that both resistant and susceptible rice varieties contained multiple Pib homologs (Wang et al. 1999). The sequence analysis of the reference genomes of Nipponbare and Shuhui498 revealed that they contain one and three copies of $\mathrm{Pib}$ homologs, respectively (Fig. 5). Moreover, nine Pib homologs were identified in different rice varieties which share significant sequence similarity to each other, indicating that the Pib locus is highly dynamic with respect to its genomic organization. The complexity of the Pib locus could blur the diagnosis of the existence of $P i b$ in different rice varieties by PCR amplification using gene-specific primers. Instead, we herein introduced a method similar to the so-called targeted amplicon sequencing (https://www.illumina.com/) by combining PCR amplification and amplicon sequencing for the diagnosis of $P i b$ in different rice varieties. Given the fact that many $R$ genes are distributed as multiple alleles or haplotypes with a high sequence similarity in different rice varieties, haplotype sequencing is believed to provide a more reliable and precise method for the diagnosis of known $R$ genes from their functional and nonfunctional alleles in different rice varieties. The distribution of $P i b$ in $>60 \%$ of released varieties in the Philippines since the late 1960s could be associated with the high selection pressure to the rice blast pathogen for the depletion of AvrPib-containing isolates in the population, which, to some extent, can explain the low resistance frequency of Pib against the contemporary blast population.

\section{LITERATURE CITED}

Böhnert, H., Fudal, I., Dioh, W., Tharreau, D., Notteghem, J., and Lebrun, M. 2004. A putative polyketide synthase/peptide synthetase from Magnaporthe grisea signals pathogen attack to resistant rice. Plant Cell 16:2499-2513.

Cesari, S., Thilliez, G., Ribot, C., Chalvon, V., Michel, C., Jauneau, A., Rivas, S., Alaux, L., Kanzaki, H., Okuyama, Y., Morel, J. B., Fournier, E., Tharreau, D., Terauchi, R., and Kroj, T. 2013. The rice resistance protein pair RGA4/RGA5 recognizes the Magnaporthe oryzae effectors AVR-Pia and AVR1-CO39 by direct binding. Plant Cell 25:1463-1481.

Chen, C., Chen, M., Hu, J., Zhang, W., Zhong, Z., Jia, Y., Allaux, L., Fournier, E., Tharreau, D., Wang, G. L., Wang, Z., Shen, W. C., Lu, G., Wang, B., and Mitchell, T. K. 2014. Sequence variation and recognition specificity of the avirulence gene AvrPiz-t in Magnaporthe oryzae field populations. Fungal Genomics Biol. 4:13.

Chen, X., Shang, J., Chen, D., Lei, C., Zou, Y., Zhai, W., Liu, G., Xu, J., Ling, Z., Cao, G., Ma, B., Wang, Y., Zhao, X., Li, S., and Zhu, L. 2006. A $\mathrm{B}$-Lectin receptor kinase gene conferring rice blast resistance. Plant J. 46:794-804

Chuma, I., Isobe, C., Hotta, Y., Ibaragi, K., Futamata, N., Kusaba, M., Yoshida, K., Terauch, R., Fujita, Y., Nakayashiki, H., Valent, B., and Tosa, Y. 2011. Multiple translocation of the Avr-Pita effector gene among chromosomes of the rice blast fungus Magnaporthe oryzae and related species. PLoS Pathog 7:e1002147

Fukuoka, S., Mizobuchi, R., Saka, N., Suprun, I., Matsumoto, T., Okuno, K., and Yano, M. 2012. A multiple gene complex on rice chromosome 4 is involved in durable resistance to rice blast. Theor. Appl. Genet. 125:551-559.

Fukuoka, S., Saka, N., Koga, H., Ono, K., Shimizu, T., Ebana, K., Hayashi, N., Takahashi, A., Hirochika, H., Okuno, K., and Yano, M. 2009. Loss of function of a proline-containing protein confers durable disease resistance in rice. Science 325:998-1001.

Fukuta, Y., Koga, I., Ung, T., Sathya, K., Kawasaki-Tanaka, A., Koide, Y., Kobayashi, N., Obara, M., Yadana, H., and Hayashi, N. 2014. Pathogenicity of rice blast (Pyricularia oryzae Cavara) isolates from Cambodia. Jpn. Agric. Res. Q. 48:155-166.

George, M. L. C., Nelson, R., Zeigler, R., and Leung, H. 1998. Rapid population analysis of Magnaporthe grisea by using rep-PCR and endogenous repetitive DNA sequences. Phytopathology 88:223-229.

IRRI. 2014. Pages 18-19 in: Standard Evaluation System for Rice, 5th ed. SES, Los Baños, Philippines.

Jones, J. D. G., and Dangl, J. L. 2006. The plant immune system. Nature 444: 323-329.

Kamoun, S. 2007. Groovy times: Filamentous pathogen effectors revealed. Curr. Opin. Plant Biol. 10:358-365.
Kang, S., Lebrun, M. H., Farrall, L., and Valent, B. 2001. Gain of virulence caused by insertion of a Pot3 transposon in a Magnaporthe grisea avirulence gene. Mol. Plant-Microbe Interact. 14:671-674.

Kanzaki, H., Yoshida, K., Saitoh, H., Fujisaki, K., Hirabuchi, A., Alaux, L., Fournier, E., Tharreau, D., and Terauchi, R. 2012. Arms race co-evolution of Magnaporthe oryzae AVR-Pik and rice Pik genes driven by their physical interactions. Plant J. 72:894-907.

Kawasaki-Tanaka, A., Hayashi, N., Yanagihara, S., and Fukuta, Y. 2016. Diversity and distribution of rice blast (Pyricularia oryzae cavara) races in Japan. Plant Dis. 100:816-823.

Khush, G. S., and Jena, K. 2009. Current status and future prospects for research on blast resistance in rice (Oryza sativa L.). Pages 1-10 in: Advances in Genetics, Genomics and Control of Rice Blast Disease. G. L. Wang and B. Valent, eds. Springer, Dordrecht, The Netherlands.

Levy, M., Correa, F. J., Zeigler, R. S., Xu, S., and Hamer, J. E. 1993. Genetic diversity of the rice blast fungus in a disease nursery in Colombia. Phytopathology 83:1427-1433.

Levy, M., Romao, J., Marchetti, M. A., and Hamer, J. E. 1991. DNA fingerprinting with dispersed repeated sequence resolves pathotypic diversity in the rice blast fungus. Plant Cell 3:95-102.

Li, W., Wang, B. H., Zhang, X., Zhang, Z., Wu, J., Hu, Y., Zhao, Q., Zhang, H., Wang, Z., Wang, G. L., Lu, G., Han, B., Wang, Z., and Zhou, B. 2009. The Magnaporthe oryzae avirulence gene AvrPiz-t encodes a predicted secreted protein that triggers the immunity in rice mediated by the blast resistance gene Piz-t. Mol. Plant-Microbe Interact. 22:411-420.

Orbach, M. J., Farrall, L., Sweigard, J. A., Chumley, F. G., and Valent, B. 2000. A telomeric avirulence gene determines efficacy for the rice blast resistance gene Pi-ta. Plant Cell 12:2019-2032.

Park, C. H., Chen, S., Shirsekar, G., Zhou, B., Khang, C. H., Songkumarn, P., Afzal, A. J., Ning, Y., Wang, R., Bellizzi, M., Valent, B., and Wang, G. L. 2012. The Magnaporthe oryzae effector AvrPiz-t targets the RING E3 ubiquitin ligase APIP6 to suppress pathogen-associated molecular pattern-triggered immunity in rice. Plant Cell 24:4748-4762.

Park, C. H., Shirsekar, G., Bellizzi, M., Chen, S., Songkumarn, P., Xie, X., Shi, X., Ning, Y., Zhou, B., Suttiviriya, P., Wang, M., Umemura, K., and Wang, G. L. 2016. The E3 ligase APIP10 connects the effector AvrPiz-t to the NLR receptor Piz-t in rice. PLoS Pathog 12:e1005529.

Saghai-Maroof, M. A., Soliman, K. M., Jorgensen, R. A., and Allard, R. W. 1984. Ribosomal DNA spacer-length polymorphisms in barley: Mendelian inheritance, chromosomal location, and population dynamics. Proc. Natl. Acad. Sci. USA 81:8014-8018.

Saleh, D., Xu, P., Shen, Y., Li, C., Adreit, H., Milazzo, J., Ravigné, V., Bazin, E., Nottéghem, J. L., Fournier, E., and Tharreau, D. 2012. Sex at the origin: An Asian population of the rice blast fungus Magnaporthe oryzae reproduces sexually. Mol. Ecol. 21:1330-1344.

Shan, W., Cao, M., Leung, D., and Tyler, B. 2004. The Avrlb locus of Phytophthora sojae encodes an elicitor and a regulator required for avirulence on soybean plants carrying resistance gene Rpslb. Mol. Plant-Microbe Interact. 17:394-403.

Stahl, E., Dwyer, A. G., Mauricio, R., Kreitman, M., and Bergelson, J. 1999. Dynamics of disease resistance polymorphism at the Rpm1 locus of Arabidopsis. Nature 400:667-671

Stergiopoulos, I., de Kock, M. J. D., Lindhout, P., and de Wit, P. J. G. M. 2007. Allelic variation in the effector genes of the tomato pathogen Cladosporium fulvum reveals different modes of adaptive evolution. Mol. Plant-Microbe Interact. 20:1271-1283

Su, J., Wang, W., Han, J., Chen, S., Wang, C., Zeng, L., Feng, A., Yang, J., Zhou, B., and Zhu, X. 2015. Functional divergence of duplicated genes results in a novel blast resistance gene Pi50 at the Pi2/9 locus. Theor. Appl. Genet. 128:2213-2225.

Talbot, N. J. 2003. On the trail of a cereal killer: Exploring the biology of Magnaporthe grisea. Annu. Rev. Microbiol. 57:177-202.

Tanweer, F., Raafi, M., Sijam, K., Rahim, H., Ahmed, F., Ashkani, S., and Latif, M. 2015. Introgression of blast resistance genes (putative $P i-b$ and $P i$ $k h$ ) into elite rice cultivar MR219 through marker-assisted selection. Front. Plant Sci. 6:1002.

Telebanco-Yanoria, M. J., Ohsawa, R., Senoo, S., Kobayashi, N., and Fukuta, Y. 2008. Diversity analysis for resistance of rice (Oryza sativa L.) to blast disease Magnaporthe grisea (Hebert) Barr. using differential isolates from the Philippines. Plant Breed. 127:355-363.

Valent, B., and Khang, C. H. 2010. Recent advances in rice blast effector research. Curr. Opin. Plant Biol. 13:434-441.

Wang, Z., Yano, M., Yamanouchi, U., Iwamoto, M., Monna, L., Hayasaka, H., Katayose, Y., and Sasaki, T. 1999. The Pib gene for rice blast resistance belongs to the nucleotide binding and leucine-rich repeat class of plant disease resistance genes. Plant J. 19:55-64.

Woolhouse, M. E., Webster, J. P., Domingo, E., Charlesworth, B., and Levin, B. R. 2002. Biological and biomedical implications of the co-evolution of pathogens and their hosts. Nat. Genet. 32:569-577. 
Wu, J., Kou, Y., Bao, J., Li, Y., Tang, M., Zhu, X., Ponaya, A., Xiao, G., Li, J., Li, C., Song, M. Y., Cumagun, R., Deng, Q., Lu, G., Jeon, J. S., Naqvi, N., and Zhou, B. 2015. Comparative genomics identifies the Magnaporthe oryzae avirulence effector $\mathrm{AvrPi} i$ that triggers $P i 9$-mediated blast resistance in rice. New Phytol. 206:1463-1475.

Xing, J., Jia, Y., Peng, Z., Shi, Y., He, Q., Shu, F., Zhang, W., Zhang, Z., and Deng, H. 2017. Characterization of molecular identity and pathogenicity of rice blast fungus in Hunan province of China. Plant Dis. 101:557-561.

Yoshida, K., Saitoh, H., Fujisawa, S., Kanzaki, H., Matsumura, H., Yoshida, K., Tosa, Y., Chuma, I., Takano, Y., Win, J., Kamoun, S., and Terauchi, K. 2009. Association genetics reveals three novel avirulence genes from the rice blast fungal pathogen Magnaporthe oryzae. Plant Cell 21: 1573-1591.

Zeigler, R., Scott, P., Leung, H., Bordeos, A., Kumar, J., and Nelson, R. 1997. Evidence of parasexual exchange of DNA in the rice blast fungus challenges its exclusive clonality. Phytopathology 87:284-294.

Zhang, S., Wang, L., Wu, W., He, L., Yang, X., and Pan, Q. 2015. Function and evolution of Magnaporthe oryzae avirulence gene AvrPib responding to the rice blast resistance gene Pib. Sci. Rep. 5: Article 11642.

Zhou, E., Jia, Y., Singh, P., Correll, J. C., and Lee, F. N. 2007. Instability of the Magnaporthe oryzae avirulence gene Avr-Pita alters virulence. Fungal Genet. Biol. 44:1024-1034. 